\title{
Atazanavir-Induced Severe Episodes of Kidney Stones in an HIV-1-Infected Subject Characterized by a CYP3A Poor Metabolizer Phenotype
}

\author{
Stefano Rusconi ${ }^{{ }^{*}}$, Roberta Gagliardini ${ }^{2}$, Felicia Stefania Falvella ${ }^{3}$, \\ Dario Cattaneo ${ }^{3}$, Valentina Di Cristo ${ }^{1}$, Andrea De Luca ${ }^{4}$, \\ Massimiliano Fabbiani ${ }^{2}$, Emilio Clementi ${ }^{5,6}$, \\ Massimo Galli ${ }^{1}$ and Simona Di Giambenedetto ${ }^{2}$ \\ ${ }^{1}$ Infectious Diseases Unit, Department of Biomedical and Clinical Sciences "Luigi Sacco", \\ Università degli Studi di Milano, Milan, Italy. \\ ${ }^{2}$ Institute of Clinical Infectious Diseases, Catholic University of the Sacred Heart, Rome, \\ Italy. \\ ${ }^{3}$ Unit of Clinical Pharmacology, Department of Biomedical and Clinical Sciences \\ "Luigi Sacco", Università degli Studi di Milano, Milan, Italy. \\ ${ }^{4}$ University Division of Infectious Diseases, University of Siena, Siena, Italy. \\ ${ }^{5}$ Scientific Institute, IRCCS Eugenio Medea, Bosisio Parini, Lecco, Italy. \\ ${ }^{6}$ Unit of Clinical Pharmacology, CNR Institute of Neuroscience, Department of Biomedical \\ and Clinical Sciences "Luigi Sacco", Università degli Studi di Milano, 20157 Milan, Italy.
}

\section{Authors' contributions}

This work was carried out in collaboration between all authors. All authors read and approved the final manuscript.

Article Information

DOI: $10.9734 / B J P R / 2014 / 14348$ Editor(s):

(1) Abdelwahab Omri, Dept. of Chemistry and Biochemistry and Dept. of Biomolecular Sciences, Laurentian Univ., Canada. Reviewers:

(1) Anonymous, University of Benin, Nigeria. (2) Antoinette van der Kuyl, Dept. of Medical Microbiology, Academic Medical Center, Univ. of Amsterdam, Amsterdam, The Netherlands. Complete Peer review History: http://www.sciencedomain.org/review-history.php?iid=789\&id=14\&aid=6954

Case Study

Received $27^{\text {th }}$ September 2014 Accepted 22 $2^{\text {nd }}$ October 2014 Published $15^{\text {th }}$ November 2014

${ }^{*}$ Corresponding author: Email: stefano.rusconi@unimi.it; 


\section{ABSTRACT}

Background: Atazanavir, an antiretroviral drug of the protease inhibitor class, is coadministered with ritonavir to inhibit atazanavir metabolism and decrease pharmacokinetic variability. Atazanavir is metabolised mainly by CYP3A4/5 enzymes. High CYP3A intersubject variability has been documented in most cases on a genetic basis. The CYP $3 A 5^{\star} 3$ allele affects splicing defect and protein truncation. Recently a new intronic variant, CYP3A4*22, was found associated with reduced CYP3A4 activity. To assess whether an altered CYP3A activity impairs the metabolism of atazanavir, we investigated the two functional polymorphisms. To the best of our knowledge, this is the first case report showing that overexposure to ATV is associated with CYP3A poor phenotype and nephrolithiasis. Further study is needed in order to confirm this interesting observation.

Case Presentation: We describe the case of a 43 year-old HIV-1-infected man treated with atazanavir/ritonavir plus lamivudine who experienced early and recurrent severe episodes of kidney stones. Atazanavir plasma trough concentrations showed a value higher than the normal range, thus we investigated the two polymorphisms that are known to affect CYP3A4/5 activity. This analysis revealed that our patient was a CYP3A poor metabolizer since he carried CYP3A $4 * 1 /{ }^{*} 22$ and CYP $3 A 5^{*} 3 /{ }^{*} 3$ genotypes.

Conclusion: We suggest that screening of CYP3A functional variants is an appropriate approach, helping in treatment choice and potential dosage adjustment of protease inhibitors.

Keywords: Atazanavir; kidney stones; HIV-1; CYP3A; HAART.

\section{BACKGROUND}

Whereas atazanavir (ATV) is well tolerated in most patients important side effects have been reported in the medium and long term [1]. In most European Countries ATV is approved only as ritonavir-boosted for the treatment of both antiretroviral naïve and experienced patients (ATV/r 300/100 mg qd). Other ways to administer ATV are formally considered as off-label.

Considerable inter-individual variability has been observed in plasma concentrations of ATV after standard dosing [2], mainly related to drug-to-drug interactions and differences in hepatic metabolism. ATV is metabolised by hepatic CYP3A4/5 enzymes to a large extent, with $20 \%$ and $7 \%$ of unmodified drug being recovered in the feces and urine, respectively [3]. Atazanavir is also an inhibitor of CYP3A and UGT1A1; coadministration of atazanavir and drugs metabolised by CYP3A or UGT1A1 may result in adverse effects [3].

Genetic bases affect CYP3A activity and two polymorphisms have been suggested to play a key role in this. The first relevant variation is CYP3A5 intron $3 A>G$ single nucleotide polymorphism (SNP) commonly known as CYP3A5*3 allele, which results in a splicing defect and protein truncation [4]; whereas the overall allele frequency of the CYP $3 A 5^{\star} 3$ allele is $31.2 \%$ it increases up to $97 \%$ in Caucasian population (1000 Genome Phase 1 genotype date). The second variation that recently has been shown to play a critical role in CYP3A4 expression and activity is CYP3A4 intron $6 \mathrm{C}>\mathrm{T}$ polymorphism, also know as CYP3A4*22 [5]; the overall allele frequency of the minor allele is $2.1 \%$ and $8 \%$ in Caucasian population (1000 Genome Phase 1 genotype date). 


\section{CASE PRESENTATION}

We report a case of a 43 years-old HIV-1-infected male patient enrolled in the AtLaS study. This is a pilot study evaluating the efficacy, safety an tolerability of treatment simplification to a dual therapy with atazanavir/ritonavir (rtv) plus lamivudine in virologically suppressed HIV+ patients [6].

The AtLaS study was conducted in accordance with the Declaration of Helsinki and all subjects signed a written informed consent.

Our patient had been receiving ATV/rtv + lamivudine + tenofovir for 4 years, had a CD4 cell count of $1,056 / \mu \mathrm{L}$, an HIV-1 RNA $<50 \mathrm{cp} / \mathrm{mL}$ and a CDC stage A. This subject had a normal renal function (i.e. serum creatinine $<1.0 \mathrm{mg} / \mathrm{dL}$ ) and bever experienced problems with kidney stones. Upon enrollment, the subject experienced early (week 8) and recurrent severe kidney stones/colics. The episodes of drug-related nephrolithiasis were diagnosed indirectly after having excluded the presence of oxalate or urate crystals. ATV plasma trough concentration was assessed by a validated high performance liquid chromatographic (HPLC) method. As shown in Table 1, ATV trough level was very high being equal to $3,190 \mathrm{ng} / \mathrm{mL}$ (therapeutic range $150-850 \mathrm{ng} / \mathrm{mL}$ ). The CYP3A5*3 and CYP3A4*22 alleles were evaluated by Real-Time PCR using LightSNiP (Roche). As expected, in Caucasian subject, the inactive CYP3A $5 * 3$ allele were found in homozygosis. Interestingly, our patient also was heterozygous for the CYP3A4*22 decrease-of-function allele, configuring a functional status of CYP3A poor metabolizer (Table 1).

Table 1. Treatment monitoring and genotyping analysis performed in our case

\begin{tabular}{|c|c|c|c|c|c|c|c|c|}
\hline $\begin{array}{l}\text { CD4 at } \\
\text { BL }(\mu L)\end{array}$ & $\begin{array}{l}\text { HIV-RNA } \\
\text { at } B L \\
(\mathrm{cp} / \mathrm{mL})\end{array}$ & Age & $\begin{array}{l}\text { CD4 } \\
\text { Nadir }\end{array}$ & BMI & $\begin{array}{l}\text { Week of } \\
\text { kidney } \\
\text { stones }\end{array}$ & $\begin{array}{l}\text { ATV } \\
\text { Trough } \\
\text { Levels* }\end{array}$ & $\begin{array}{l}\text { CYP3A4*22 rs } \\
35599367 C>T\end{array}$ & $\begin{array}{l}\text { CYP3A5*3 rs } \\
776746 A>G\end{array}$ \\
\hline 1,056 & 49 & 43 & 238 & 27,43 & 38 & 3190 & CT & GG \\
\hline
\end{tabular}

Other four out of the 40 patients enrolled in the ATLAS study became affected by kidney stones and/or clinically defined colics at a time later than our case. The ATV trough levels in these patients ranged from 1,150 to $1,900 \mathrm{ng} / \mathrm{mL}$. Pharmacogenetic testing of their DNAs detected the CYP3A4 references allele and CYP3A5*3 in homozygous status defining them as belonging to the CYP3A intermediate phenotype. In one patient, the kidney stone has been analysed and ATV presence has been confirmed (7.9\%-15.8\% ATV in the composition of the stones) [7].

\section{DISCUSSION}

Despite ATV being generally well tolerated, a higher incidence of renal stones among patients receiving ATV/rtv-containing antiretroviral therapy is reported [1]. Sometimes, cases of ATV-associated urolitiasis leading to obstructive uropathy and acute renal failure have been described [8]. Our patient experienced early severe and recurrent episodes of kidney stones. Recently, Nishijima and colleagues demonstrated that in HIV-1-infected Japanese patients, ATV-induced nephrolithiasis was associated with UGT1A-3'-UTR variants [9]. In 
this respect two considerations need to be taken into account: firstly, the glucuronidation pathway represents a minor biotransformation pathway for ATV; secondly and more importantly, the ethnicity is an important factor influencing the genetic variability of the major ATV metabolic pathway. Indeed, the Caucasian population presents almost exclusively a CYP $3 A^{*} 3 /{ }^{*} 3$ genetic background and the activity of CYP3A5 is absent. The simultaneous determination of the common CYP3A4*22 allele, absent in Japanese population (1000 Genome Phase 1 genotype date), may help to identify CYP3A poor metabolisers who can be at risk of early complications due to excessively high ATV concentration. Unmodified ATV is reported to be excreted in urine; the proportion of ATV in the urine increases and precipitation and renal stones formation may occur if ATV metabolism is reduced. To the best of our knowledge this is the first report showing that polymorphisms in CYP3A locus are likely to be involved in ATV overexposure and increase the risk of nephrolithiasis. Further studies are required to fully clarify the relationship between renal calculi induced by ATV and CYP3A genotypes. If our preliminary observation will be reconfirmed in a large series of patients receiving ATV/r based anti-HIV therapy, the identification of CYP3A4*22 and CYP $3 A^{*} 3$ alleles may help infectious disease physicians in prescribing a more adequate dose of ATV. This will prevent adverse events through the maintenance of an optimal viroimmunologic efficacy.

\section{CONCLUSION}

To the best of our knowledge this is the first report showing that polymorphisms in CYP3A locus are likely to be involved in ATV overexposure and increase the risk of nephrolithiasis. Further studies are required to fully clarify the relationship between renal calculi induced by ATV and CYP3A genotypes. If our preliminary observation will be reconfirmed in a large series of patients receiving ATV/r based anti-HIV therapy, the identification of CYP3A4*22 and $\mathrm{CYP} \mathrm{A} 5^{\star} 3$ alleles may help infectious disease physicians in prescribing a more adequate dose of ATV. This will prevent adverse events through the maintenance of an optimal viro-immunologic efficacy.

\section{CONSENT}

Written informed consent was obtained from the patient for publication of this Case report. A copy of the written consent is available for review by the Editor of this journal.

\section{ETHICAL APPROVAL}

All authors hereby declare that all experiments have been examined and approved by the appropriate ethics committee and have therefore been performed in accordance with the ethical standards laid down in the 1964 Declaration of Helsinki.

\section{COMPETING INTERESTS}

Authors have declared that no competing interests exist.

\section{REFERENCES}

1. Hamada Y, Nishijima T, Watanabe K, et al. High incidence of renal stones among HIVinfected patients on ritonavir-boosted atazanavir than in those receiving other protease inhibitor-containing antiretroviral therapy. Clin Infect Dis. 2012;55:1262-1269. 
2. Le Tiec C, Barrail A, Goujard C, Taburet AM. Clinical pharmacokinetics and summary of efficacy and tolerability of atazanavir. Clin Pharmacokinet. 2005;44:1035-1050.

3. Bristol-Myers Squibb, Princeton NJ. Reyataz (atazanavir sulfate): full prescription information (package insert); 2012.

4. Kuehl P, Zhang J, Lin Y, Lamba J, Assem M, Schuetz J, Watkins PB, Daly A, Wrighton SA, Hall SD, Maurel P, Relling M, Brimer C, Yasuda K, Venkataramanan R, Strom S, Thummel K, Boguski MS, Schuetz E. Sequence diversity in CYP3A promoters and characterization of the genetic basis of polymorphic CYP3A5 expression. Nat Genet. 2001;27:383-391.

5. Wang D, Guo Y, Wrighton SA, Cooke GE, Sadee W. Intronic polymorphism in CYP3A4 affects hepatic expression and response to statin drugs. Pharmacogenomics J. 2011;11:274-286.

6. Di Giambenedetto S, Fabbiani M, Colafigli M et al. Safety and feasibility of treatment simplification to atazanavir/ritonavir + lamivudine in HIV-infected patients on stable treatment with two nucleos $(\mathrm{t})$ ide reverse transcriptase inhibitors + atazanavir/ritonavir with virological suppression (Atazanavir and Lamivudine for treatment Simplification, AtLaS pilot study). J Antimicrob Chemother. 2013;68:1364-1372.

7. Fabbiani M, Bracciale L, Doino $M$, et al. Tenofovir discontinuation could predispose to urolithiasis in atazanavir-treated patients. J Infect. 2011;62:319-321.

8. Anderson PL, Lichtenstein KA, Gerig NE, Kiser JJ, Bushman LR. Atazanavircontaining renal calculi in an HIV-infected patient. AIDS. 2007;21:1060-1062.

9. Nishijima T, Tsuchiya K, Tanaka N, et al. Single-nucleotide polymorphisms in the UDP-glucuronosyltransferase 1A-3' untranslated region are associated with atazanavir-induced nephrolithiasis in patients with HIV-1 infection: A pharmacogenetic study. J Antimicrob Chemother; 2014. pii: dku304. [Epub ahead of print].

(0) 2014 Rusconi et al.; This is an Open Access article distributed under the terms of the Creative Commons Attribution License (http://creativecommons.org/licenses/by/3.0), which permits unrestricted use, distribution, and reproduction in any medium, provided the original work is properly cited.

Peer-review history:

The peer review history for this paper can be accessed here: http://www.sciencedomain.org/review-history.php?iid=789\&id=14\&aid=6954 